$(\mathrm{p}<0.0001)$. IMT in the aortic arch was $1.21 \pm 0.63 \mathrm{~mm}$ in SLE patients vs $0.98 \pm 0.25 \mathrm{~mm}$ in healthy controls $(\mathrm{p}=0.002)$. Areas of increased IMT showed regular wall thickening of medium echogenicity indicating possible inflammatory origin. Microcirculation as measured with mean oxygen saturation peak was decreased in SLE patients versus controls, 83.7 $\pm 7.8 \%$ vs $86.7 \pm 4.6 \%(p=0.01)$.

Conclusion This study indicates that an extended ultrasound protocol to detect possible inflammatory vessel wall changes and/or early atherosclerosis in SLE is of value. In addition we showed impaired microcirculatory function as measured with EPOS in SLE patients. Further validation of macro and microcirculatory lesions are warranted in larger studies.

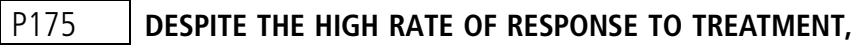 LUPUS NEPHRITIS STANDARD OF CARE IS STILL ASSOCIATED WITH HIGH INCIDENCE OF CHRONIC KIDNEY DISEASE: A RETROSPECTIVE LONGITUDINAL STUDY, FROM THREE SOUTH-EUROPEAN COHORTS OF PATIENTS IN FOLLOW-UP SINCE 2000}

${ }^{1}$ Irene Altabás, ${ }^{1} J o s e$ María Pego, ${ }^{3}$ Francisco Rubiño ${ }^{2}$ Chiara Stagnaro, ${ }^{2}$ Marta Mosca ${ }^{2}$ Íñigo Rua-Figueroa. ${ }^{1}$ Rheumatology Dept., CHUVI, Vigo, Spain; ${ }^{2}$ Dept. of Clinical and Experimental Medicine, Rheumatology Unit, University of Pisa, Italy; ${ }^{3}$ Rheumatology Dept., Hospital Doctor Negrin, Las Palmas, Spain

10.1136/lupus-2020-eurolupus.217

Background One of the most important complications of lupus nephritis (LN) is the chronic kidney disease (CKD) development.

Methods Multicenter retrospective observational study of SLE patients (ACR97) with biopsy proven $\mathrm{LN}$ attending to three South European Rheumatology departments in the last two decades. Variables: demographics; SLE-related, including global activity (SLEDAI-2K), renal flares, therapies, ACR response criteria and CKD. Statistical analysis: bivariate and multivariate analysis exploring factors associated to CKD. ROC curves and area under the curve were calculated to test each proteinuria level as predictor of long-term renal outcome.

Results Seventy-six patients were included, mean age: 33 years; mean disease duration: 14 years; mean follow-up (since LN diagnosis): 8,5 years. LN class III, IV and V were present in $22 \%, 75 \%$ and $3 \%$ of the cases, respectively. Cyclophosphamide was the most used treatment to induce remission (55\%). At 3, 6 and 12th months, the mean proteinuria was $2.3 \mathrm{~g} / 24 \mathrm{~h}, 1.53 \mathrm{~g} / 24 \mathrm{~h}, 1.1 \mathrm{~g} / 24 \mathrm{~h}$, respectively $(\mathrm{p}<0.001)$. Fifty-five $(77,5 \%)$ achieved complete response and $61(84.7 \%)$ complete or partial response. Median time to renal remission: 12.5 months (6,17.5). Sixteen (21.9\%) patients developed CKD.

In the logistic regression model, using genetic algorithms, we found that proteinuria at 6 months was significantly associated with CKD (OR:2.95; 95\%CI 1.19,9.29, $\mathrm{p}=0.03)$. Hypertension and male sex were marginally associated $(\mathrm{p}=0.06$, both). The optimal cut-off point of proteinuria at 6 months was $0.7 \mathrm{~g} /$ day, (sensitivity: $50 \%$; specificity: $93 \%)$.

Conclusions A considerable percentage of LN developed CKD. Proteinuria at 6 months was associated with CKD.
An absolute level of proteinuria below $0.7 \mathrm{~g} /$ day measured at 6 months is the best predictor of long-term renal outcomes.

\section{P176 A CASE OF LUPUS ENTERITIS SUCCESSFULLY TREATED WITH ANTI-TNF ALPHA INHIBITOR}

${ }^{1}$ Silvia Bilia, ${ }^{1}$ Daiana Giannini, ${ }^{2}$ Domenico Giannese, ${ }^{3}$ Gloria Mumolo, ${ }^{1}$ Riccardo Capecchi, ${ }^{3}$ Linda Ceccarelli, ${ }^{1}$ Paola Migliorini, ${ }^{1}$ Antonio Tavoni. ' Clinical Immunology and Allergy Unit, Dept. of Clinical and Experimental Medicine, University of Pisa, Pisa; ${ }^{2}$ Nephrology, Dialysis and Transplantation AOUP Pisa, Pisa; ${ }^{3}$ IBD Unit, Dept. of General Surgery and Gastronterology, Pisa, Italy

\subsection{6/lupus-2020-eurolupus.218}

Background Gastro-intestinal manifestations in systemic lupus erythematosus (SLE) can affect up to $40 \%$ of patients, including enteritis presenting as mesenteric vasculitis, pseudoobstruction or protein-losing enteropathy. We present a case of lupus enteritis successfully treated with anti-TNFalpha inhibitor.

Methods A 28-year-old woman was evaluated for diarrhea, abdominal pain, fever and rectal bleeding not responsive to antibiotics. She had a thirteen-year history of SLE in remission with Mycophenolate Mofetil and previous muco-cutaneous and haematologic relapses, myocarditis and end-stage renal disease (IV-class glomerulonephritis). She previously underwent multiple immunosuppressants including ciclophosphamide, cyclosporine, anti-CD20, immunoglobulins. One month before the onset of symptoms she discontinued MMF for worsening anemia. Simultaneously we reported signs of lupic flare (low C3, haemolytic anemia, lymphopenia, fever, arthralgias and malar rash). Pulse-steroids and IVIg followed by cyclosporine were initially performed with only temporary benefit. Enteric CT-scan and endoscopy revealed chronic and acute colo-rectal and gastric inflammation (cryptitis, erosions, necrosis, microgranulomas). Anti-TNFalpha inhibitor Infliximab (5 mg/kg) was added to Azathioprine $50 \mathrm{mg} /$ daily. Within a month we observed clinical and serological sustained remission.

Results Typically, mesenteric vasculitis involves small arteries or venules. Histological examination reveals submucosal and muscular layers infiltration and necrotizing vasculitis, with panmural predominant eosinophilic, neutrophilic or mixed infiltrate. The distinction of inflammatory bowel disease (IBD) from enteric-SLE can be challenging. In this case, an early anti-flogistic therapy may have led to uncomplete microscopic patterns not fullfilling criteria neither for enteric vasculitis nor IBD. A lupic flare with predominant gastro-enteric presentation is the most plausible hypothesis because of the infrequent association between SLE and IBD and simultaneous extra-intestinal lupic features. Abdominal involvement in a patient previously treated with high dosage cyclophosphamide $(10 \mathrm{~g})$ and the lack of response to azathioprine lead to the introduction of anti-TNFalpha inhibitor.

Conclusion The role of TNFalpha in SLE is controversial and TNFalpha inhibitors are reported to control SLE-arthritis. Further studies are needed to evaluate their role in the management of gastro-enteric SLE. 